\title{
Homozygous deletions of UGT2B17 modifies effects of smoking on TP53-mutations and relapse of head and neck carcinoma
}

\author{
Aki Mafune ${ }^{1,5}$, Takanori Hama ${ }^{1,2^{*}}$, Toshihito Suda ${ }^{2}$, Yutaka Suzuki ${ }^{4}$, Masahiro Ikegami ${ }^{3}$, Chikako Sakanashi ${ }^{1}$,
} Satoko Imai ${ }^{1}$, Akio Nakashima ${ }^{1,5}$, Takashi Yokoo ${ }^{5}$, Kota Wada ${ }^{2,6}$, Hiromi Kojima ${ }^{2}$ and Mitsuyoshi Urashima ${ }^{1}$

\begin{abstract}
Background: Smoking induces oncogenic TP53-mutations in head and neck squamous cell carcinomas (HNSCCs). Disruptive mutations of TP53-gene and expression of p16 protein [p16 (+)] in tumor tissue associate with worse and better prognosis, respectively. UDP-glucuronosyltransferase 2 family, polypeptide B17 (UGT2B17) detoxifies smoking-related metabolites. Differences among ethnic groups in UGT2B17 are extremely high. Homozygous deletions of UGT2B17 gene (UGT2B17-deletion) are a common copy number variant (CNV) among Japanese, but not a common CNV among Africans and Europeans. Thus, we examined Japanese patients with HNSCC to explore if UGT2B17-deletion and/or p16 (+) modify effects of smoking on TP53-mutations and affect relapse.

Methods: We conducted a posthoc analysis of a prospective cohort. Polymerase chain reaction, immunohistochemistry, and direct sequencing were used to determine UGT2B17-deletion, p16 (+), and detailed TP53-mutations, respectively.

Results: UGT2B17-deletion was observed in $80 \%$ of this study population. For this $80 \%$, TP53-mutations were significantly more common among smokers than non-smokers $(P=0.0016)$, but this difference between smokers and nonsmokers was not significant for the 20\% with UGT2B17. In patients with UGT2B17-deletion and $p 16(+)$, simultaneously, TP53-mutations were much more common among smokers than among non-smokers ( $81 \%$ versus $17 \% ; P=0.0050$ ). Patients with both UGT2B17-deletion and disruptive TP53-mutations had higher relapse rates than other patients (hazard ratio, 2.22; 95\% confidence interval, 1.30 to 3.80, $P=0.004$ ) in a stepwise method.

Conclusions: These results suggest that UGT2B17-deletion interacting with p16 (+) may modify effects of smoking on TP53-mutations and may further interact with the disruptive TP53-mutations to raise relapse rates among Japanese patients with HNSCC.
\end{abstract}

Keywords: UGT2B17, TP53, HNSCC (head and neck squamous-cell carcinoma) and smoking

\section{Background}

Tobacco smoking is associated with 5 million deaths per year worldwide and is regarded as one of the leading causes of premature death [1]. Nicotine, a natural ingredient in tobacco leaves, is so addictive that people smoke habitually, which in turn results in exposure to a diverse array of carcinogens. Metabolites of nicotine, including cotinine and other compounds, are further catabolized and detoxified

\footnotetext{
* Correspondence: takanori@jikei.ac.jp

'Division of Molecular Epidemiology, Jikei University School of Medicine, Tokyo, Japan

${ }^{2}$ Department of Oto-Rhino-laryngology, Jikei University School of Medicine, 3 - 25 - 8 Nishi-shimbashi, Minato-ku, Tokyo 105-8461, Japan Full list of author information is available at the end of the article
}

via CYP2A6 [2] and the UDP-glucuronosyltransferase (UGT) family of enzymes. One UGT gene, UDPglucuronosyltransferase 2 family, polypeptide B17 (UGT2B17) enzyme decreases the abundance nicotinerelated metabolites via glucuronidation [3]. Consequently, UGT2B17 gene deletions may reduce detoxification rates of carcinogens in tobacco and tobacco smoke [4]. Therefore, this UGT2B17-deletion may increase an individual's susceptibility to tobacco-related cancers, e.g., lung cancer [5].

Copy number variants (CNVs) of UGT2B17 gene, known to vary greatly among ethnic populations; for example, homozygous deletion of UGT2B17 (0 copy) is not a 
common CNV among Africans or Europeans e.g., 14\% of Nigerians, but it is common among East Asian populations, e.g., $92 \%$ of Japanese [6]. Smoking is a major risk factor for head and neck squamous cell carcinoma (HNSCC) [7], by inducing oncogenic mutations of the TP53 oncosuppressor gene [8] and of other genes [9,10]. In particular, disruptive mutations in TP53 were associated with reduced survival in patients with HNSCC [11].

Therefore, we hypothesized that smoking may increase the risk of TP53-mutations among patients with homozygous for UGT2B17 deletions (defined as "UGT2B17deletion" in this study) to a greater extent than among patients with one or two copies of UGT2B17 (defined as "UGT2B17-presence" in this study). Because UGT2B17 deletion is common among Japanese, the power to detect interacting effects between smoking and UGT2B17deletion on TP53-mutations can be enhanced by focusing on Japanese patients with HNSCC. In addition to TP53-mutations, overexpression of $p 16$-protein [defined as " $p 16(+)$ " in this study] in tumors, which is encoded by $C D K N 2 A$, increases survival time in cases of oropharyngeal cancer $[12,13]$. We reported that heavy alcohol consumption triggered previously known and unknown somatic copy number alterations (SCNAs) including CDKN2A, but that smoking induced TP53-mutations [14]. Using this cohort of Japanese patients with HNSCC as post hoc analysis, we newly explored if UGT2B17-deletion modify effects of smoking on TP53-mutations, in combination with $p 16(+)$. Furthermore, we studied if combinations among UGT2B17-deletion, p16 (+), and disruptive TP53-mutations affect cancer relapse.

\section{Methods}

\section{Study design}

We conducted a cohort study at Jikei University Hospital from March 2006 to November 2012. The study protocol was reviewed and approved by the Ethics Committee for Biomedical Research of the Jikei Institutional Review Board. The entire process of study design, data monitoring, and data analyses were performed in the Division of Molecular Epidemiology. Eligible participants were Japanese patients with HNSCC (oropharyngeal, hypopharyngeal, laryngeal, oral and nasal cancer) aged 20 years or older who had newly diagnosed or recurrent disease. A total of 262 patients provided written informed consent to participate in this study. Of these 262 patients, 28 patients were excluded because pathological diagnosis was not squamous cell carcinoma or because the primary tumor site was unknown. 27patient received in combination with chemotherapy or radiotherapy after surgery for close surgical margin and/or extracapsular spread of metastatic node. All of them were stage IV. Clinical data from the remaining 234 patients were used. Clinical information was obtained from clinical and surgical charts. Tumor node metastasis (TNM) classification and cancer stages were determined according to the $6^{\text {th }}$ Union for International Cancer Control TNM classification and stage groupings. Tumor grade with regard to cell differentiation was classified into three categories-well differentiated, moderately differentiated, or poorly differentiated-by a pathologist (M.I.). Of these 234 patients, nine patients were unknown of cell differentiation.

\section{Smoking and alcohol drinking}

A history of current or past cigarette smoking was obtained based on a questionnaire completed by each patient at surgery. The age at which they started smoking and the number of cigarettes smoked per day was recorded. For past smokers, the age at which the patient ceased smoking was also recorded. The extent of previous smoking was quantified in pack-years (PYs); 10 PYs is any equivalent to smoking 1 pack including 20 cigarettes/day for 10 years (e.g., 2 packs/day for 5 years). Patients were classified as smokers if they had smoked at for least 10 PYs within the 20 years preceding diagnosis of HNSCC. Non-smokers were defined as patients who had never smoked, had not smoked in the 20 years preceding diagnosis, or smoked less than 10 PY prior to surgical resection of HNSCC. Of these 234 patients, two patients were unknown of smoking status.

The following three categories were used to classify patients based upon average daily alcohol consumption during the 20 years preceding diagnosis of HNSCC: 1 ) non-drinkers were defined as patients who did not consume alcohol or consumed less than one drink per day; 2) moderate drinkers were defined as patients who consumed at least one, but less than two, drinks per day, and 3) heavy drinkers were defined as patients who consumed two or more drinks per day. One drink was defined as containing approximately $10 \mathrm{~g}$ of alcohol, which is equal to $30 \mathrm{ml}$ of hard liquor, $100 \mathrm{ml}$ of wine containing $12 \%$ alcohol, or $360 \mathrm{ml}$ of beer.

\section{Samples}

With each patient's consent, peripheral blood samples and tumor tissue were collected during the operation. QIAamp DNA Micro Kits 50 (Qiagen, Tokyo, Japan) were used to purify extracted DNA, and NanoVue plus (General Electric healthcare Japan, Tokyo, Japan) was used to measure DNA concentration in each sample; samples were then frozen at $-80^{\circ} \mathrm{C}$ until use.

\section{Array-based comparative genome hybridization (CGH)}

An Agilent Enzymatic Labeling Kit was used according to the manufacturer's instructions to label $0.5 \mu \mathrm{g}$ of genomic DNA for each CGH array. Labeled DNA was hybridized to an Agilent-022060 SurePrint G3 Human CGH Microarray 4x180K (Agilent Technologies, Inc., Santa 
Clara, CA, USA); the Agilent Microarray Scanner and Feature Extraction v.10.7.3.1 (Agilent Technologies), were used according to manufacturer's instruction to scan probed arrays. Control DNA was obtained from one Japanese individual who is an author (MU) of this study. We focused only on previously reported SCNAs of CDKN2A [14] and on CNVs of UGT2B17 that are associated with metabolism of nicotine [15]. The data described in this article have been deposited in NCBIs Gene Expression Omnibus (GEO) [16] and are accessible through GEO series accession number GSE47443.

\section{TaqMan Real-time PCR}

We also performed real-time polymerase chain reaction (PCR) to confirm the microarray data. The TaqMan-based real-time PCR method for comparative quantification was performed with extracted DNA according to Life Technologies' protocol. Genomic sequences of UGT2B17 were used to generate the specific target sequence. Primers for UGT2B17 (Taqman Copy Number Assays No. 186891217) and a probe for RNase P (Taqman copy number Reference Assay RNase P No. 4401631) were used (Life Technologies Corp.). Reactions $(20 \mu \mathrm{L})$ were performed in 96-well plates using Brilliant III Ultra-Fast QPCR Master Mix, Reference Dye (30 nM), nuclease-free water $(8 \mu \mathrm{L})$, DNA sample $(1 \mu \mathrm{L})$, and $U G T 2 B 17$ primer $(1 \mu \mathrm{L})$ (Applied Biosystems) or TaqMan Copy Number Reference Assay RNase $P(1 \mu \mathrm{L})$; reaction mixtures were subjected to 40 cycles of $95^{\circ} \mathrm{C}$ for $3 \mathrm{~min}, 95^{\circ} \mathrm{C}$ for $10 \mathrm{~s}$, and $60^{\circ} \mathrm{C}$ for $30 \mathrm{~s}$. For the precise and accurate amplification of DNA, each assay with each primer pairs was run in duplicate. Comparative quantification was calculated using a sample from the same person (MU) who provided the control samples for the CGH array. A MX 3005P Real-Time QPCR System with Mx Pro Software version 4.10 (Agilent Technologies) was used to measure the product of each real-time PCR assay.

The method of measurement was based on the comparative cycle threshold $(\mathrm{Ct})$ method for the target sequence (UGT2B17) and a reference sequence (RNase P). The RNase $P$ gene was co-amplified with $U G T 2 B 17$ and served as an internal standard. The PCR amplification efficiencies of RNase P and UGT2B17 were $100 \%$ and 99\%; these were calculated by using the comparative $\Delta \Delta \mathrm{Ct}$ methods as described by Pfaffl et al. [17]. The fold changes in copy numbers of the gene were $\log 2$ transformed and determined to be gene positive or gene negative (over two copies or not). Finally, 97\% of array results were consistent with real-time PCR.

\section{PCR to differentiate between one and two copies of UGT2B17}

In $3 \%$ of samples, array and real-time PCR results were conflicted and could not differentiate between one and two copies of the UGT2B17 gene, To determine the absence or presence of the UGT2B17 gene, we further performed PCR as follows. Because a high level of sequence identity exists between the UGT2B17 and UGT2B15 genes, we used gene-specific PCR primers to distinguish UGT2B17 from UGT2B15 and to distinguish between one and two copies of the UGT2B17 gene: Marker D (Forward primer 5'-TCACAAGTCAATCTCCCATCC-3', Reverse primer 5'-CTGCAGAATATGTCAATAATTGG C-3') is positive for one copy and two copies (100 bp), Marker J (Forward primer 5'-TGCACAGAGTTAAGA AATGGAGAGATGTG-3', Reverse primer 5'-GATCAT CCTATATCCTGACAGAATT-3') is positive for only one copy (900 bp) $[18,19]$. PCR reactions were carried out in $25-\mu \mathrm{l}$ mixtures containing $1 \mu \mathrm{g}$ of genomic DNA, $2.5 \mu \mathrm{L}$ of $10 x \mathrm{xA}$ PCR buffer II, $2 \mu \mathrm{L}$ of dNTP $(400 \mu \mathrm{M})$, $0.25 \mu \mathrm{L}$ of LA Taq (Takara Bio Inc., Shiga, Japan), 18.25 $\mu \mathrm{L}$ of nuclease-free water, and $0.5 \mu \mathrm{L}$ of each of the two primers $(100 \mathrm{pmol} / \mu \mathrm{L})$. Each reaction mixture was incubated at $94^{\circ} \mathrm{C}$ for $3 \mathrm{~min}$ and then subjected to 30 cycles of $94^{\circ} \mathrm{C}$ for $20 \mathrm{~s}, 60^{\circ} \mathrm{C}$ for $30 \mathrm{~s}$, and $72^{\circ} \mathrm{C}$ for $90 \mathrm{~s}$; each reaction was then incubated at $16^{\circ} \mathrm{C}$ until analysis.

\section{TP53-mutations}

The quality or quantity of DNA samples from 14 patients was not adequate to assess TP53 mutational status; therefore, only 234 samples were analyzed with regard to TP53-mutations.

Exons 2 thru 11 of the TP53 gene were each independently amplified by PCR using purchased primers following the manufacturer's protocol (NIPPON GENE Co. Ltd., Chiyoda-ku, Tokyo, Japan). Each resulting PCR product was cloned and then sequenced with the $\mathrm{ABI}$ PRISM 3700 Genetic Analyzer (Life Technologies Corp.). The following 10 single-nucleotide polymorphisms V31I, P36P, P47S, P72R, R158R, R213R, V217M, P222P, T312S, and G360A-are reportedly each caused by a single nucleotide polymorphism [20], and thus excluded from total TP53-mutations. Disruptive TP53-mutations were defined as non-conservative mutations located inside the key DNA-binding domain (L2-L3 region) or as stop codons in any region [9]. Sites containing cytidine phosphate guanosine $(\mathrm{CpG})$ dinucleotides were determined according to the database of WHO's International Agency for Research on Cancer and based on the work by Petitjean et al. [21].

\section{p16 immunohistochemistry}

Formalin-fixed, paraffin-embedded tumor specimens were evaluated for $p 16$ overexpression with a rabbit monoclonal antibody that recognizes $p 16$ (Anti-CDKN2A/p16INK4a antibody [EPR1473]: Abcam Plc, Science Park, Cambridge, England). In this study, positive $p 16$-protein expression 
(designated $p 16(+)$ ) determined via immunohistochemistry (IHC) was defined as strong and diffuse nuclear, cytoplasmic staining or both in at least $70 \%$ of tumor cells. Any other pattern of $p 16$ expression was classified as $p 16(-)$.

\section{Statistical analysis}

To evaluate significant differences between groups, the unpaired $t$ test and the Mann-Whitney test were used to analyze ages and PYs, respectively. The chi-square test was used to assess categorical variables. Interaction effects between smoking and each of ten sub-groupingsage ( $<$ vs. $\geq 65$ years), gender, drinking status, primary sites of tumor, tumor grades, stages, UGT2B17-CNV, CDKN2A-SCNA, $p 16-\mathrm{ICH}$, and UGT2B17-CNV and p16-ICH combined-were assessed with respect to any type of TP53-mutations; potential interactions were assessed by a $P_{\text {interaction }}$ term. Then, for each sub-grouping, risks for any kind of TP53-mutations were compared between smokers and non-smokers using a risk ratio (RR) with a 95\% confidence interval (95\% CI).

In survival analyses, the time from surgery to relapse was used to calculate relapse-free rates. Patients were considered as "censored", when follow-ups were stopped at the time of a patient's death by causes other than HNSCC relapse or the last outpatient clinic visit. The Cox proportional hazard model was used to calculate each hazard ratio (HR) with a 95\% CI. To distinguish significant prognostic factors from non-significant factors, a stepwise backward elimination method was applied to all 13 factors identified-age, gender, smoker (10PYs $\leq$ ), heavy drinker, primary sites of tumor, CDKN2A-SCNA, p16-ICH, disruptive TP53-mutations, UGT2B17-deletion, interaction between disruptive TP53-mutations and UGT2B17-deletion, interaction between disruptive TP53mutations and $p 16(+)$, stages, tumor grades - with a cutoff point of $P=0.05$. The Kaplan-Meier survival curves were drawn based on relapse-free rates; log-rank tests were used to compare these rates differentiated by p16 (+), UGT2B17-deletion and disruptive TP53mutations. Each $\mathrm{P}<0.05$ was considered statistically significant. However, the Bonferroni correction was used to correct for multiple testing, and each pairwise interaction among the 10 subgroups was considered significant when $P_{\text {interaction }}$ was less than 0.005. All statistical analyses were performed using STATA 13.1 (STATA Crop., College Station, TX).

\section{Results}

Patient characteristics

Patient characteristics were compared between nonsmokers and smokers and between patients with wild-type TP53 and those with any type of TP53-mutations in the primary tumors (Table 1). Tumors with TP53-mutations were significantly more common among smokers (67\%) than among non-smokers (52\%) (RR: $1.29,95 \%$ CI: 1.00 to $1.65, P=0.030)$, which we have already reported [14]. Men $(P<0.001)$ and alcohol-drinkers $(P<0.001)$ were also significantly more common among smokers than among non-smokers. Oral cancer was more frequent among nonsmokers than smokers compared with other primary tumor sites $(P=0.030)$. Well differentiated histology was less common among smokers than non-smokers. Heterozygous and homozygous deletions of the CDNK2A-gene were significantly more prevalent among patients with TP53-mutations than those with wild-type TP53 ( $P=$ 0.035). Additionally, we found that $80 \%$ of this study population harbored UGT2B17-deletions. However, nonsmokers did not differ significantly from smokers with regard to p16 (+) or UGT2B17-CNVs; similarly, patients with wild-type TP53 did not differ significantly from those with TP53-mutations with regard to p16 (+) or UGT2B17CNVs.

Then, we focused more closely on TP53 status of tumors. Of the 234 tumor samples analyzed, 86 samples had no TP53 mutation, 84 had one mutation, 27 had two mutations, 20 had three, 7 had four, 9 had five, and 1 had six. The frequencies of specific base-pair changes among these 234 patients were as follows: A:T > C:G, 1 (0.4\%); A:T > G:C, 13 (5.6\%), A:T > T:A, 5 (2\%); G:C > A:T, 60 (26\%); G:C > C:G, 19 (8\%); G:C > T:A, 82 (35\%). The frequencies of other types of mutations were as follows: deletion, 10 (4\%); insertion, 4 (2\%); nonsense, 63 (27\%); missense, 69 (30\%); frameshift, 14 (6\%). In non-smokers, 9 in 37 (24\%; 95\% CI, 12 to 41\%) TP53-mutations occurred at CpG sites, but in smokers, 13 in 108 (12\%; 95\% CI, 7 to 20\%) did.

\section{Effects modifiers of smoking on TP53-mutations}

Interactions between smoking and each of 11 variablesage, gender, alcohol drinking status, the primary sites of tumors, tumor grades, stages, the number of lymph node metastasis, UGT2B17-deletion, CDKN2A-SCNAs, p16 $(+)$, and a combination of UGT2B17-deletion and $p 16$ $(+)$-were assessed (Table 2). In variables of the primary sites of tumors, CDKN2A-SCNAs, $p 16(+)$, and a combination of UGT2B17-deletion and p16 (+), interactions were analyzed except for HPV-positive patients. Smoking interacted significantly with four factors-stages, UGT2B17-deletion, $p 16(+)$, and the combination of UGT2B17-deletions and p16 (+)-to induce TP53mutations, but not with age $(P=0.55)$, gender $(P=0.22)$, drinking status $(P=0.90)$, primary tumor sites $(P=0.09)$, tumor grades $(P=0.30)$, the number of lymph node metastasis $(P=0.51)$ or CDKN2A-SCNAs $(P=0.08)$. Restricting to patients with UGT2B17-deletion, TP53-mutations were more prevalent among smokers than among nonsmokers $(P=0.0016)$, but restricting to patients with UGT2B17-presence, differences between smokers and 
Table 1 Patient ${ }^{* 1}$ characteristics assessed based on smoking status and TP53-mutations

\begin{tabular}{|c|c|c|c|c|c|c|c|}
\hline & Total & $\begin{array}{l}\text { Smokers }^{* 2} \\
(\mathrm{~N}=161: 69 \%)\end{array}$ & $\begin{array}{l}\text { Non-smokers }^{* 2} \\
(\mathrm{~N}=71: 31 \%)\end{array}$ & p-value & $\begin{array}{l}\text { Mutant TP53 } \\
(\mathrm{N}=147: 63 \%)\end{array}$ & $\begin{array}{l}\text { Wild-type } \\
\text { TP53 } \\
\text { (N=87: } 37 \%)\end{array}$ & p-value \\
\hline \multicolumn{8}{|l|}{ Smoking status - PYs } \\
\hline $25 \% / 50 \% / 75 \%$ & $0 / 25 / 40$ & $25 / 40 / 46$ & 0/0/0 & $<0.0001^{* 3}$ & $8 / 30 / 40$ & $0 / 20 / 40$ & $0.085^{* 3}$ \\
\hline Smokers - no. (\%) & $161(69)$ & - & - & - & $108(74)$ & $53(61)$ & $0.030^{* 4}$ \\
\hline TP53-mutations - no. (\%) & $147(63)$ & $108(67)^{* 7}$ & $37(52)^{* 7}$ & $0.030^{* 4}$ & & & \\
\hline Age, years - yr. mean \pm s.d. & $63.2 \pm 10.9$ & $63.5 \pm 10.2$ & $62.6 \pm 12.6$ & $0.56^{* 5}$ & $64.1 \pm 10.4$ & $61.6 \pm 11.5$ & $0.082^{* 5}$ \\
\hline Men - no. $(\%)^{*}$ & $187(80)$ & $152(94)$ & $33(46)$ & $<0.001^{* 4}$ & $122(83)$ & $65(75)$ & $0.13^{* 4}$ \\
\hline Drinking status - no. $(\%)^{* 6}$ & & & & $<0.001^{* 4}$ & & & $0.043^{* 4}$ \\
\hline Non-drinker & $89(38)$ & $35(22)$ & $53(75)$ & & $50(34)$ & $39(45)$ & \\
\hline Moderate drinker & $74(32)$ & $64(40)$ & $9(13)$ & & $55(37)$ & $19(22)$ & \\
\hline Heavy drinker & $71(30)$ & $62(39)$ & $9(13)$ & & $42(29)$ & $29(33)$ & \\
\hline Primary site of tumor - no. $(\%)^{*}$ & & & & $0.030^{* 4}$ & & & $0.13^{* 4}$ \\
\hline Oropharyngea & $63(27)$ & $47(29)$ & $16(23)$ & & $37(25)$ & $26(30)$ & \\
\hline Hypopharyngeal & $64(27)$ & $47(29)$ & $16(23)$ & & $49(33)$ & $15(17)$ & \\
\hline Laryngeal & $29(12)$ & $24(15)$ & $5(7)$ & & $17(12)$ & $12(14)$ & \\
\hline Oral & $57(24)$ & $32(20)$ & $25(35)$ & & $32(22)$ & $25(29)$ & \\
\hline Nasal & $21(9)$ & $11(7)$ & $9(13)$ & & $12(8)$ & $9(10)$ & \\
\hline Cell differentiation - no. $(\%)^{* 6}$ & & & & $0.023^{* 4}$ & & & $0.94^{* 4}$ \\
\hline Well differentiated & $69(31)$ & $39(25)$ & $29(43)$ & & $45(31)$ & $24(29)$ & \\
\hline Moderately differentiated & $111(49)$ & $84(54)$ & $26(39)$ & & $70(49)$ & $41(50)$ & \\
\hline Poorly differentiated & $45(20)$ & $33(21)$ & $12(18)$ & & $28(20)$ & $17(21)$ & \\
\hline Stages - no. $(\%)^{* 6}$ & & & & $0.12^{* 4}$ & & & $0.97^{* 4}$ \\
\hline | & $12(5)$ & $11(7)$ & $1(1)$ & & $8(5)$ & $4(5)$ & \\
\hline$\|$ & $48(21)$ & $34(21)$ & $14(20)$ & & $29(20)$ & $19(22)$ & \\
\hline III & $48(21)$ & $28(18)$ & $20(29)$ & & $30(21)$ & $18(21)$ & \\
\hline IV & $124(53)$ & $87(54)$ & $35(50)$ & & $79(54)$ & $45(52)$ & \\
\hline \multicolumn{8}{|l|}{ Anticancer therapy - no. (\%) } \\
\hline Radiotherapy \pm Chemotherapy & $27(12)$ & $22(13)$ & $4(6)$ & $0.13^{* 4}$ & $15(10)$ & $12(14)$ & $0.41^{* 4}$ \\
\hline CDKN2A-SCNAs - no. $(\%)^{*}$ & & & & $0.73^{* 4}$ & & & $0.035^{* 4}$ \\
\hline Norma & $174(77)$ & $117(76)$ & $55(80)$ & & $99(72)$ & $75(86)$ & \\
\hline Heterozygous deletion & $39(17)$ & $29(19)$ & $10(14)$ & & $29(21)$ & $10(11)$ & \\
\hline Homozygous deletion & $12(5)$ & $8(5)$ & $4(6)$ & & $10(7)$ & $2(2)$ & \\
\hline p16 (+) - no. (\%) & $47(20)$ & $28(17)$ & $19(27)$ & $0.10^{* 4}$ & $24(16)$ & $23(26)$ & $0.062^{* 4}$ \\
\hline UGT2B17 CNVs - no. $(\%)^{*}$ & & & & $0.60^{* 4}$ & & & $0.95^{* 4}$ \\
\hline Homozygous deletions: 0 copy & $181(80)$ & $124(81)$ & $55(80)$ & & $111(80)$ & $70(80)$ & \\
\hline Heterozygous deletion: 1 copy & $42(19)$ & $28(18)$ & $14(20)$ & & $26(19)$ & $16(18)$ & \\
\hline Normal: 2 copies & $2(1)$ & $2(1)$ & $0(0)$ & $0.60^{* 4}$ & $1(1)$ & $1(1)$ & $0.95^{* 4}$ \\
\hline
\end{tabular}

${ }^{*}$ S 5 moking history was unavailable for two of the 234 .

${ }^{*}$ Non-smokers were defined as having a $<10-\mathrm{PYs}$ history; smokers were defined as having a $\geq 10-\mathrm{PY}$ s history.

${ }^{*}$ Mann-Whitney test was used to calculate the $p$-value.

${ }^{*} X^{2}$ test was used to calculate the $p$-value. ${ }^{* 5}$ Student's $t$ test was used to calculate the -value.

${ }^{*}$ Because of rounding, total values are not always $100 \% .{ }^{* 7} \mathrm{RR}, 1.29 ; 95 \% \mathrm{Cl}, 1.00$ to 1.65 .

non-smokers were not significant. Similarly, restricting to patients with $p 16(+)$ tumors, smoking increased the risk of TP53-mutations up to 3.48-fold in comparison with non-smoking, but not in restricting to patients with $p 16$ (-) tumors. In restricting to patients with UGT2B17deletion and had $p 16(+)$, smokers had significantly higher 
Table 2 Effects modifiers of smoking on TP53-mutations in tumors ${ }^{* 1}$

\begin{tabular}{|c|c|c|c|c|c|c|}
\hline Outcome: any type of TP53-mutations & $P_{\text {interaction }}{ }^{* 2}$ & Smokers N = 167 & Non-smokers $\mathrm{N}=65$ & RR & $95 \% \mathrm{Cl}$ & p-value \\
\hline Primary sites of tumor - no. $(\%)^{*} 6$ & 0.09 & & & & & \\
\hline Oropharyngeal & & $26(76)$ & $4(80)$ & 0.96 & 0.59 to 1.54 & 0.86 \\
\hline Hypopharyngeal & & $37(79)$ & $11(69)$ & 1.15 & 0.80 to 1.64 & 0.42 \\
\hline Laryngeal & & $14(58)$ & $3(60)$ & 0.97 & 0.44 to 2.15 & 0.95 \\
\hline Oral & & $15(48)$ & $16(67)$ & 0.73 & 0.46 to 1.15 & 0.18 \\
\hline Nasal & & $10(91)$ & $1(11)$ & 8.18 & 1.28 to 52.4 & 0.0004 \\
\hline Stages - no. (\%) & 0.0019 & & & & & \\
\hline । & & $7(64)$ & $1(100)$ & 0.64 & 0.41 to 0.99 & 0.46 \\
\hline$\|$ & & $19(56)$ & $10(71)$ & 0.78 & 0.50 to 1.22 & 0.32 \\
\hline III & & $21(75)$ & $9(45)$ & 1.67 & 0.98 to 2.83 & 0.034 \\
\hline IV & & $60(69)$ & $17(49)$ & 1.42 & 0.98 to 2.05 & 0.035 \\
\hline UGT2B17 CNVs ${ }^{* 5}-$ no. (\%) & 0.0016 & & & & & \\
\hline UGT2B17-deletion: 0 copy & & $85(69)$ & $24(44)$ & 1.57 & 1.14 to 2.17 & 0.0016 \\
\hline UGT2B17-presence: 1 copy or 2 copies & & $16(53)$ & $11(79)$ & 0.68 & 0.44 to 1.05 & 0.11 \\
\hline CDKN2A SCNA ${ }^{* 3^{* 6}}-$ no. $(\%)$ & 0.08 & & & & & \\
\hline Normal: 2 copies & & $68(65)$ & $22(51)$ & 1.27 & 0.91 to 1.75 & 0.12 \\
\hline Heterozygous deletion: 1 copy & & $21(78)$ & $7(70)$ & 1.11 & 0.71 to 1.75 & 0.62 \\
\hline Homozygous deletions: 0 copy & & $6(75)$ & $4(100)$ & 0.75 & 0.50 to 1.12 & 0.27 \\
\hline p16-ICH in tumor ${ }^{* * 6}-$ no. (\%) & 0.043 & & & & & \\
\hline p16 (-) & & $85(68)$ & $33(66)$ & 1.03 & 0.82 to 1.30 & 0.80 \\
\hline p16 (+) & & $17(77)$ & $2(22)$ & 3.48 & 1.00 to 12.1 & 0.0043 \\
\hline UGT2B17-CNVs \& p16-ICH - no. (\%) & 0.0080 & & & & & \\
\hline UGT2B17-presence \& p16 (-) & & $13(57)$ & $10(91)$ & 0.62 & 0.42 to 0.93 & 0.045 \\
\hline UGT2B17-deletions \& p16 (-) & & $67(69)$ & $21(57)$ & 1.22 & 0.89 to 1.66 & 0.18 \\
\hline UGT2B17-presence \& p16 (+) & & $2(50)$ & $1(33)$ & 1.50 & 0.23 to 9.80 & 0.66 \\
\hline UGT2B17-deletions \& p16 (+) & & $13(81)$ & $1(17)$ & 4.88 & 0.80 to 29.6 & 0.0050 \\
\hline
\end{tabular}

${ }^{*}$ Any type of TP53-mutations observed in the tumor genome was used as the outcome.

${ }^{*} \mathrm{P}_{\text {interaction }}$ was calculated as interaction effect between a factor and smoking on the risk of TP53-mutations in a tumor. With the Bonferroni correction, $\mathrm{p}<0.005$ was considered as statistically significant.

${ }^{*}$ CDKN2A SCNA: Copy number alterations of CDKN2A, which encodes $p 16$, were determined by CGH array.

${ }^{*} \mathrm{p} 16$ overexpression in tumor samples was determined via $\mathrm{ICH}$ and classified as positive (+) or negative (-).

${ }^{*}$ UGT2B17 CNV: Copy number variants of UGT2B17 were screened via CGH array and confirmed by real-time PCR and PCR to differentiate between one and two copies of UGT2B17-gene.

${ }^{*}$ Interaction was analyzed except for HPV-positive patients.

risk of TP53-mutations than did non-smokers (RR, 4.88; 95\% CI, 0.80 to $29.6 ; P=0.0050)$, but not in other combinations: UGT2B17-presence and p16 (-), UGT2B17deletion and $p 16(-)$, and UGT2B17 presence and $p 16$ (+) (Table 2).

\section{Prognostic factors}

Using backward elimination for 13 candidate prognostic factors (Table 3), we found that disruptive TP53-mutations and UGT2B17-deletion interacted to significantly increase the risk of relapse (HR, 2.22; 95\% CI, 1.30 to $3.80, P=$ 0.004); however, either TP53-mutations or UGT2B17deletion alone did not significantly affect the risk. Notably, p16 (+) was a better prognostic factor than p16 (-) (HR, 0.53 ; $95 \% \mathrm{CI}, 0.29$ to $0.99, P=0.047)$. Thus, we analyzed three grouping of the 234 patients based on combinations of three factors $-p 16(+)$ tumors, presence of disruptive TP53-mutations, and UGT2B17-deletions. During a median follow-up period of 1.5 years (interquartile range, 1.0 to 2.5 years), relapse occurred in 89 of 232 patients (38\%) in this study. Based on Kaplan-Meier curves, patients harboring both UGT2B17-deletion and a disruptive TP53mutation in the primary tumors had the highest relapse rates among the three groups, and the group comprising patients with $p 16(+)$ tumors and lacking any disruptive TP53-mutation in the primary tumors had the lowest relapse rates (Figure 1). Relapse was occurred in 21 of 35 patients in the group of both UGT2B17-deletion and a disruptive TP53-mutation in the primary tumors (indicated as green-colored line in Figure 1), 16 of 59 patients 
Table 3 Cox proportional hazard model as determined with backward eliminated via a stepwise method $^{* 1}$

\begin{tabular}{llll}
\hline Outcome: any type of TP53-mutations in a tumor & HR & $\mathbf{9 5 \%} \mathrm{Cl}$ & \multicolumn{1}{c}{-value } \\
\hline Having both disruptive TP53-mutations and UGT2B17-deletions & 2.22 & 1.30 to 3.80 & 0.004 \\
p16-positive tumor & 0.53 & 0.29 to 0.99 & 0.047 \\
Stage IV & 2.32 & 1.44 to 3.74 & 0.001 \\
Poorly differentiated tumor grade & 1.66 & 1.01 to 2.74 & 0.047 \\
\hline
\end{tabular}

${ }^{* 1}$ By backward elimination from age, gender, smoker (10 PYs $\leq$ ), heavy drinker, primary site of tumor, CDKN2A-SCNAs, p16 (+), disruptive TP53-mutations, UGT2B17-deletion, interaction effect between disruptive TP53-mutations and UGT2B17-deletion, interaction between disruptive TP53-mutations and p16 (+), stages, tumor grade of cell differentiation.

in the group of both $p 16(+)$ and no disruptive TP53mutation in the primary tumors (indicated as bluecolored line in Figure 1) and 48 of 131 patients in the other groups (indicated as red-colored line in Figure 1). We also analyzed overall survival by Kaplan-Meier curves. Although patients with $p 16(+)$ tumors and lacking any disruptive TP53-mutation in the primary tumors had the highest survival rates than the other groups $(P=0.0190$, figure was not shown), there was no significant effect among these three factors; status of disruptive TP53mutation, p16 and UGT2B17.

\section{Discussion}

The prevalence of copy number variants (CNVs) of UGT2B17 gene is quite different among ethnic populations. The frequency of UGT2B17-deletion was only about 10 to $15 \%$ among general Caucasian population or Caucasian with lung cancer [5,22]. In contrast, the frequency of UGT2B17-deletion among Japanese athletes was $74.5 \%$ in male and $60.2 \%$ in female [23] and $92 \%$ among those contributing to the Japanese HapMap [6]. We first confirmed that homozygous deletion of UGT2B17 is highly prevalent among this cohort of Japanese patients. Of the 234 patients examined, $80 \%$ were homozygous for UGT2B17-deletions; $19 \%$ carried one copy of $U G T 2 B 17$, and only $1 \%$ had two copies; these findings were within the range of previous reports for the Japanese or East Asian [6,23]. Therefore, Japanese patients with HNSCC constitute a valid study population for examination of the influence of UGT2B17deletion on TP53 mutation ratio and relapse rates.

Log-rank test: $P=0.0102$

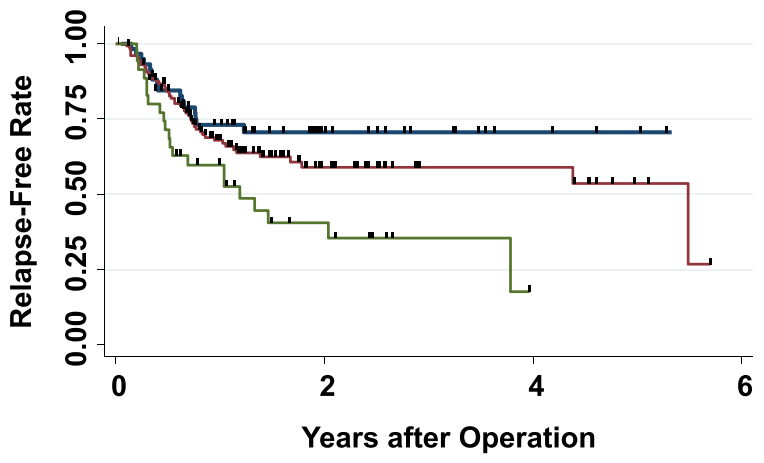

\begin{tabular}{|c|c|c|c|}
\hline 工 & \multicolumn{2}{|c|}{ p16 (+) - dTP53 (-) - UGT2B17 (-) } & or p16 (+) - dTP53 (-) - UGT2B17 (+) \\
\hline No. at risk & 59 & 43 & 43 \\
\hline & \multicolumn{2}{|c|}{$[$ p16 (-) - dTP53 (-) - UGT2B17 (-) } & or p16 (-) - dTP53 (+) - UGT2B17 (+) \\
\hline & \multicolumn{2}{|c|}{$[$ p16 (+) - dTP53 (+) - UGT2B17 (+) } & or p16 (-) - dTP53 (-) - UGT2B17 (+) \\
\hline No. at risk & 131 & 85 & 85 \\
\hline & \multicolumn{2}{|c|}{ p16 (-) - dTP53 (+) - UGT2B17 (-) } & or p16 (+) - dTP53 (+) - UGT2B17 (-) \\
\hline No. at risk & 35 & 16 & 14 \\
\hline
\end{tabular}

Figure 1 Kaplan-Meier curves of relapse-free rates in 234 patients with HNSCC. Differences in time until relapse were compared among combinations of 16 (+) tumors, disruptive TP53-mutations, and homozygous UGT2B17 deletions. The group of both UGT2B17-deletion and a disruptive TP53-mutation in the primary tumors is indicated as green-colored line, the group of both p16 (+) and no disruptive TP53-mutation in the primary tumors is indicated as blue-colored line and the other groups are indicated as red-colored line. p16 (+): p16-positive tumor; p16 (-): p16- negative tumor; dTP53 (+): presence of disruptive TP53-mutations; dTP53 (-): no disruptive TP53-mutations or wild-type TP53; UGT2B17 (+): UGT2B17-deletion: homozygous deletion of UGT2B17; UGT2B17 (-): UGT2B17-presence: one or two copies of UGT2B17. 
We next found that $63 \%$ of all tumors had some type of mutation within exon-2 to exon-11 of TP53. There were significantly more TP53-mutant tumors among smokers than non-smokers, which we have already reported [14]. Moreover, the frequency of TP53-mutations at $\mathrm{CpG}$ sites was 2-fold higher among non-smokers than among smokers. These findings were consistent with previous findings from whole-exome sequencing studies [9].

Thirdly, we found a significant interaction effect between UGT2B17-deletion and smoking on TP53 mutation rate $\left(P_{\text {interaction }}=0.0016\right)$. Specifically, restricting to patients with UGT2B17-deletion, TP53-mutations were significantly more common in tumors from smokers than those from non-smokers, but not for patients with UGT2B17-presence; to our knowledge, these and following findings have not been reported previously. UGT2B17deletion may 1) reduce a person's ability to detoxify smoking-associated metabolites, 2) allow cellular DNA to become exposed to high levels of carcinogens in cigarettes and cigarette smoke, and 3) raise the probability of DNA injury, and thus increase the risk of TP53-mutations, resulting in tumor development. Moreover, p16 expression also interacted with smoking to increase the risk of TP53-mutations. Specifically, restricting to patients with p16 (+) tumors, the frequency of TP53-mutations was 3.48-fold higher among smokers than non-smokers. Overexpression of $p 16$ can result from disruption of the negative feedback loop that normally operates among p16, cyclin-dependent kinases, cyclins, and phosphorylation of retinoblastoma protein; several causes-including human papillomavirus infection-can disrupt this loop [24], and such disruption may facilitate cell cycle progression and make cells more susceptible to the carcinogens in cigarettes and cigarette smoke.

We then assessed a combination of these two variablesUGT2B17-deletion and $p 16(+)$-to assess potential interactions between them. Restricting to patients with UGT2B17-deletion and p16 (+) tumors, TP53-mutations were significantly more common among tumors from smokers $(81 \%)$ than those from non-smokers (17\%) (RR, 4.88; $95 \% \mathrm{CI}, 0.80$ to $29.6 ; P=0.0050)$. On the other hand, patients with UGT2B17-presence and $p 16(-)$ tumors, TP53-mutations were significantly less common among tumors from smokers (57\%) than those from non-smokers (91\%) (RR, 0.62; 95\% CI, 0.42 to 0.93; $P=0.045$ ). From these findings, together UGT2B17-deletion and p16 (+) synergistically enhanced the risk of TP53-mutations occurring in tumors, because UGT2B17-deletion reduced the metabolism and detoxification of metabolites from cigarette smoke and $p 16$-overexpression reflected abnormal cell cycle progression and increased cellular susceptibility to carcinogens.

In survival analyses, we confirmed that patients survival were significantly associated with stages, cell differentiation levels and the number of lymph node metastasis before adjustment; these results were consistent with previous studies. Then we analyzed using stepwise elimination and survival analysis by adjusting for stages, cell differentiation levels and others.

Fourth, we found a significant interaction between disruptive TP53-mutations and UGT2B17-deletion. Patients with TP53-mutant tumors and UGT2B17-deletion were more than twice as likely to relapse as all other patients; this finding was novel and striking. In contrast, patients with $p 16(+)$ tumors and wild-type TP53 were half as likely to relapse as those with other patterns of tumor mutation; this finding was consistent with a previous finding about oropharyngeal cancer [12].

There were four main limitations in this study. Only two patients had two copies of UGT2B17; therefore, we mainly compared the effects of homozygosity with those of heterozygosity with regard to UGT2B17-deletions. Among 262 participants, TP53-mutations could not be measured in 28 samples, because of too small size of resected tumors to use for this study. Third is we analyzed relapse-free survival within the patients who had newly diagnosed or recurrent disease. It appears that one of the causes for no significant differences in the over survival may be the effect by the curative treatment to recurrence. Fourth is the most patients were advanced stage III to IV (74\%) in this study. In spite of limited to the patients with early stage I to II, the patients harboring both UGT2B17-deletion and a disruptive TP53-mutation in the primary tumors had the highest relapse rates among the three groups using Kaplain-Meier curves (Log-rank test, $\mathrm{P}=0.0071$, figure was not shown).

\section{Conclusions}

In conclusions, homozygous UGT2B17-deletion may interact with smoking and $p 16$-protein expression to increase the risk of TP53-mutations, and may further interact with disruptive TP53-mutations to raise relapse rates among Japanese patients with HNSCC.

\footnotetext{
Abbreviation

HNSCC: Head and neck squamous cell carcinoma; UGT2B17: UDPglucuronosyltransferase 2 family, polypeptide B17; CNV: Common copy number variant; UGT: UDP-glucuronosyltransferase; SCNA: Somatic copy number alterations; TNM: Tumor node metastasis; PY: Pack-year; CGH: comparative genome hybridization; PCR: Polymerase chain reaction; CpG: Cytidine phosphate guanosine; IHC: Immunohistochemistry; RR: Risk ratio; 95\% Cl: 95\% confidence interval; HR: Hazard ratio.

\section{Competing interests}

The authors declare that they have no competing interests.

Authors' contributions

YS, TH,TK, HK and MU designed the study. TH KWand TS contributed to collecting the tissue samples and clinical data. MI contributed to pathological examination. AM, CS, SI and AN have carried out molecular studies. AM and MU performed analysis and interpretation of data. AM, TH and $\mathrm{MU}$ participate in drafting the manuscript. All authors have read and approved the final manuscript.
} 


\section{Acknowledgements}

We would also like to thank Mr. Hiroaki Suga and Mr. Takeshi Mimura for arranging samples. This research was supported by the Ministry of Education, Culture, Sports, Science and Technology in the Japan-Supported Program for the Strategic Research Foundation at Private Universities the Ministry of Education, Science, Sports and Culture, Grant-in-Aid for Scientific Research (C) and The Jikei University Research Fund. All authors read and approved the final manuscript.

\section{Author details}

${ }^{1}$ Division of Molecular Epidemiology, Jikei University School of Medicine, Tokyo, Japan. ${ }^{2}$ Department of Oto-Rhino-laryngology, Jikei University School of Medicine, 3 - 25 - 8 Nishi-shimbashi, Minato-ku, Tokyo 105-8461, Japan. ${ }^{3}$ Department of Pathology, Jikei University School of Medicine, Tokyo, Japan. ${ }^{4}$ Department of Surgery, International University of Health and welfare, Tochigi, Japan. ${ }^{5}$ Division of Nephrology and Hypertension, Department of Internal Medicine, Jikei University School of Medicine, Tokyo, Japan. ${ }^{6}$ Department of Otorhinolaryngology, Toho University, Tokyo, Japan.

Received: 19 October 2014 Accepted: 19 March 2015

Published online: 31 March 2015

\section{References}

1. Hatsukami DK, Stead LF, Gupta PC. Tobacco addiction. Lancet. 2008:371:2027-38

2. Hukkanen J, Jacob 3rd P, Benowitz NL. Metabolism and disposition kinetics of nicotine. Pharmacol Rev. 2005;57:79-115.

3. Caldwell WS, Greene JM, Byrd GD, Chang KM, Uhrig MS, de Bethizy JD, et al Characterization of the glucuronide conjugate of cotinine: a previously unidentified major metabolite of nicotine in smokers' urine. Chem Res Toxicol. 1992;5:280-5.

4. Lazarus P, Zheng Y, Runkle EA, Muscat JE, Wiener D. Genotype-phenotype correlation between the polymorphic UGT2B17-gene deletions and NNAL glucuronidation activities in human liver microsomes. Pharmacogenet Genomics. 2005;15:769-78

5. Gallagher CJ, Muscat JE, Hicks AN, Zheng Y, Dyer AM, Chase GA, et al. The UDP-glucuronosyltransferase 2B17 gene deletions polymorphism: sex-specific association with urinary 4-(methylnitrosamino)-1-(3-pyridyl)-1-butanol glucuronidation phenotype and risk for lung cancer. Cancer Epidemiol Biomarkers Prev. 2007;16:823-8.

6. Xue Y, Sun D, Daly A, Yang F, Zhou X, Zhao M, et al. Adaptive evolution of UGT2B17 copy-number variation. Am J Hum Genet. 2008;83:337-46.

7. LLubin JH, Purdue M, Kelsey K, Zhang ZF, Winn D, Wei Q, et al. Tota exposure and exposure rate effects for alcohol and smoking and risk of head and neck cancer: a pooled analysis of case-control studies. Am Epidemiol. 2009;170:937-47.

8. Brennan JA, Boyle JO, Koch WM, Goodman SN, Hurban RH, Eby YJ, et al. Association between cigarette smoking and mutation of the p53 gene in squamous-cell carcinoma of the head and neck. N Engl J Med. 1995;332:712-7.

9. Stransky N, Egloff AM, Tward AD, Kostic AD, Cibulskis K, Sivachenko A, et al. The mutational landscape of head and neck squamous cell carcinoma. Science. 2011;333:1157-60.

10. Ang KK, Harris J, Wheeler R, Weber R, Rosenthal DI, Nguyen-Tân PF, et al Exome sequencing of head and neck squamous cell carcinoma reveals inactivating mutations in NOTCH1. Science. 2011:333:1154-7.

11. Poeta ML, Manola J, Goldwasser MA, Forastiere A, Benoit N, Califano JA, et al. TP53-mutations and survival in squamous-cell carcinoma of the head and neck. N Engl J Med. 2007;357:2552-61.

12. Rischin D, Young RJ, Fisher R, Fox SB, Le QT, Peters $L$, et al. Prognostic significance of p16INK4A and human papillomavirus in patients with oropharyngeal cancer treated on TROG 02.02 phase III trial. J Clin Oncol. 2010;28:4142-8.

13. Ang KK, Harris J, Wheeler R, Weber R, Rosenthal DI, Nguyen-Tân PF, et al. Human papillomavirus and survival of patients with oropharyngeal cancer. N Engl J Med. 2010;363:24-35.

14. Urashima M, Hama T, Suda T, Suzuki Y, Ikegami M, Sakanashi C, et al. Distinct effects of alcohol consumption and smoking on genetic alterations in head and neck carcinoma. PLoS One. 2013;8:e80828.
15. Chen G, Giambrone Jr NE, Dluzen DF, Muscat JE, Berg A, Gallagher CJ, et al. Glucuronidation genotypes and nicotine metabolic phenotypes: importance of functional UGT2B10 and UGT2B17 polymorphisms. Cancer Res. 2010;70:7543-52.

16. NCBls Gene Expression Omnibus (GEO). [http://www.ncbi.nlm.nhi.gov/geo/]

17. Pfaffl MW. A new mathematical model for relative quantification in real-time RT-PCR. Nucleic Acids Res. 2001;29(9):e45.

18. Yang TL, Chen XD, Guo Y, Lei SF, Wang JT, Zhou Q, et al. Genome-wide copy-number-variation study identified a susceptibility gene, UGT2B17, for osteoporosis. Am J Hum Genet. 2008;83:663-74.

19. Chew S, Mullin BH, Lewis JR, Spector TD, Prince RL, Wilson SG. Homozygous deletion of the UGT2B17 gene is not associated with osteoporosis risk in elderly Caucasian women. Osteoporos Int. 2011:22:1981-6.

20. International Agency for Research on Cancer (IARC) TP53 Database. [http://p53.iarc.fr/TP53GeneVariations.aspx]

21. Petitjean A, Mathe E, Kato S, Ishioka C, Tavtigian SV, Hainaut P, et al. Impact of mutant p53 functional properties on TP53 mutation patterns and tumo phenotype: lessons from recent developments in the IARC TP53 database. Hum Mutat. 2007;28:622-9.

22. Gruber M, Le T, Filipits M, Gsur A, Mannhalter C, Jäger U, et al. UDP-glucuronosyltransferase 2B17 genotype and the risk of lung cancer among Austrian Caucasians. Cancer Epidemiol. 2013;37:625-8.

23. Okano M, Ueda T, Nishitani Y, Kano H, Ikekita A, Kageyama S. UDP-glucuronosyltransferase 2B17 genotyping in Japanese athletes and evaluation of the current sports drug testing for detecting testosterone misuse. Drug Test Anal. 2013;5:166-81.

24. Dyson N, Howley PM, Münger K, Harlow E. The human papilloma virus-16 E7 oncoprotein is able to bind to the retinoblastoma gene product. Science. 1989;243(4893):934-7.

\section{Submit your next manuscript to BioMed Central and take full advantage of:}

- Convenient online submission

- Thorough peer review

- No space constraints or color figure charges

- Immediate publication on acceptance

- Inclusion in PubMed, CAS, Scopus and Google Scholar

- Research which is freely available for redistribution

Submit your manuscript at www.biomedcentral.com/submit 